\title{
Inflammation-related epigenetic risk and child and adolescent mental health: A prospective study from pregnancy to middle adolescence
}

\author{
EDWARD D. BARKER, ${ }^{a}$ CHARLOTTE A. M. CECIL, ${ }^{a}$ ESTHER WALTON,${ }^{b}$ LOTTE C. HOUTEPEN, ${ }^{b}$ \\ THOMAS G. O'CONNOR, ${ }^{c}$ ANDREA DANESE, ${ }^{a}$ SARA R. JAFFEE, ${ }^{d}$ SARAH K. G. JENSEN, ${ }^{e}$ \\ CARMINE PARIANTE, ${ }^{a}$ WENDY MCARDLE, ${ }^{b}$ TOM R. GAUNT, ${ }^{b}$ CAROLINE L. RELTON, ${ }^{b}$ \\ AND SUSANNA ROBERTS ${ }^{a}$ \\ ${ }^{a}$ King's College London; ${ }^{b}$ University of Bristol; ${ }^{c}$ University of Rochester Medical Center; ${ }^{d}$ University of Pennsylvania; and \\ ${ }^{e}$ Harvard University Medical School
}

\begin{abstract}
In 785 mother-child (50\% male) pairs from a longitudinal epidemiological birth cohort, we investigated associations between inflammation-related epigenetic polygenic risk scores (i-ePGS), environmental exposures, cognitive function, and child and adolescent internalizing and externalizing problems. We examined prenatal and postnatal effects. For externalizing problems, one prenatal effect was found: i-ePGS at birth associated with higher externalizing problems (ages 7-15) indirectly through lower cognitive function (age 7). For internalizing problems, we identified two effects. For a prenatal effect, i-ePGS at birth associated with higher internalizing symptoms via continuity in i-ePGS at age 7. For a postnatal effect, higher postnatal adversity exposure (birth through age 7) associated with higher internalizing problems (ages 7-15) via higher i-ePGS (age 7). Hence, externalizing problems were related mainly to prenatal effects involving lower cognitive function, whereas internalizing problems appeared related to both prenatal and postnatal effects. The present study supports a link between i-ePGS and child and adolescent mental health.
\end{abstract}

Children who experience adversities (e.g., poverty, caregiver psychopathology, low social support, and harsh parenting) are at risk of developing persisting stress-related psychiatric disorders that can span externalizing and internalizing problems (Barker, Walton, \& Cecil, in press). Research has examined multiple possible mediating mechanisms for these associations; stress physiology has received the bulk of this

We are extremely grateful to all the families who took part in this study, the midwives for their help in recruiting them, and the whole ALSPAC team, which includes interviewers, computer and laboratory technicians, clerical workers, research scientists, volunteers, managers, receptionists, and nurses. With regard to the ALSPAC DNA methylation, we thank all involved, particularly the laboratory scientists and bioinformaticians who contributed considerable time and expertise to the data in this paper. The UK Medical Research Council and the Wellcome Trust (Grant102215/2/13/2) and the University of Bristol provide core support for ALSPAC. ARIES was funded by BBSRC Grants BBI025751/1 and BB/I025263/1. ARIES is maintained under the auspices of the MRC Integrative Epidemiology Unit at the University of Bristol (MC_UU_12013/2 and MC_UU_12013/8). This research was specifically supported by National Institute of Child and Human Development Grant R01HD068437 (to E.D.B.), Economic and Social Research Council Grants ES/R005516/1 (to E.D.B.) and ES/N001273/1 (to C.C.), and ESRC/BBSRC Grant ES/N000382/1 (to L.C.H.) for the Interpreting epigenetic signatures in studies of early life adversity project (Interstela Project).

Address correspondence and reprint requests to: Edward D. Barker, Department of Psychology, King's College London, Institute of Psychiatry, Psychology and Neuroscience, De Crespigny Park, London, SE5 8AF UK; E-mail: ted.barker@kcl.ac.uk. attention (O'connor, Moynihan, \& Caserta, 2014). In the current study we consider an alternative mechanism, namely, inflammation (Dantzer, O'Connor, Freund, Johnson, \& Kelley, 2008). The "hidden wounds hypothesis" (Danese \& Baldwin, 2017; Miller \& Chen, 2013) proposes that early psychosocial adversity translates into biological risk for mental illness by affecting the regulation of the immune system at sensitive stages of brain development (Danese \& Lewis, 2016; Nusslock \& Miller, 2016). Recent evidence suggests that DNA methylation, a type of epigenetic modification that regulates gene expression, may be a biological mechanism by which adversity can result in low-grade inflammation (McDade et al., 2017). However, little is known about how adversity in different developmental periods may affect immune-related DNA methylation, which could affect neurodevelopmental and mental health problems. This study examines the concerted influences of pre- and postnatal adversity, an immune-related DNA methylation biomarker, and cognitive function (a proxy for brain function) on the development of mental health problems from childhood to adolescence.

\section{Prenatal Adversity, Inflammation, and Cognitive Function}

During the prenatal period, larger immune molecules (e.g., cytokines and antibodies) may have access to the developing 
fetal brain via the blood-brain barrier (BBB; Goasdoué, Miller, Colditz, \& Bjorkman, 2016). There is good evidence in animal studies, and increasing evidence in humans, that inflammation in the mother during pregnancy can affect several highly orchestrated, and hence vulnerable, aspects of fetal brain development (Knuesel et al., 2014). A possible mechanism for brain and behavioral abnormalities following maternal immune activation includes the downstream effects of cytokines and other mediators of inflammation (e.g., glucocorticoids) on neural plasticity. Here, cytokines and antibodies interact with various neural and nonneural cell types (e.g., microglia) and play an important role in brain developmental processes, such as synapse modeling, neurogenesis, and neurotransmission that underlie cognitive and behavioral development (Danese \& Lewis, 2016).

In humans, maternal immune activation is associated with increased serum levels of pro-inflammatory cytokines (Andersson et al., 2016; Coussons-Read, Okun, \& Nettles, 2007). Higher levels of inflammatory makers, such as C-reactive protein (CRP) at birth (cord blood), a sensitive marker of low-grade chronic inflammation, can signpost a range of obstetric risks including amniotic fluid infection (Yoon et al., 2000), neonatal sepsis (Joram et al., 2006), and predict neurologic impairment in preterm infants (Kaukola et al., 2006). Furthermore, a study reported that placental indicators of inflammation in preterm newborns associated with variation in messenger RNA expression of genes that, in turn, predicted cognitive impairment at 10 years of age (Tilley et al., 2017). As animal models show that maternal immune activation can associate with prenatal adversity (Babri, Doosti, \& Salari, 2014), these data may be consistent with a wide range of studies that have highlighted the relationship of prenatal adversity with subsequent impaired child cognitive function (Barker, Kirkham, Ng, \& Jensen, 2013; Jensen, Dumontheil, $\&$ Barker, 2014). Of note, variation in cognitive function has been linked to both internalizing and externalizing problems (Rock, Roiser, Riedel, \& Blackwell, 2014; Séguin, Nagin, Assaad, \& Tremblay, 2004).

\section{Inflammation and Cognitive Function}

After birth, peripheral inflammation can induce neuroinflammation (Dantzer et al., 2008) and modulate the continued development and function of the brain (Danese \& Lewis, 2016). There are several pathways that link the peripheral and the brain immune systems (Dantzer et al., 2008). Peripheral cytokines can stimulate afferent fibers of the vagus nerve, which in turn can stimulate cytokine production within the brain. Cytokines can also cross into the brain where the BBB is permeable or leaky, or engage surface receptors on astrocytes and endothelial cells that form the BBB. Animal research suggests that the immune system modulates synaptogenesis, synaptic refinement, and survival, as well as myelination via its role as a modulator of oligodendrocyte function (Danese \& Lewis, 2016; Nusslock \& Miller, 2016). Moreover, animal research suggests that early life immune activation can bias microglia (central nervous system resident macrophage cells) toward an aggressive inflammatory response and reduce glucocorticoid sensitivity (Frank, Hershman, Weber, Watkins, \& Maier, 2014).

In humans, adversity-related infection and low-grade chronic inflammation are associated with impaired child cognitive function and language (Jiang et al., 2017; Patrick et al., 2005), as well as an increased risk for developing depression in adolescence and adulthood (Danese et al., 2009, 2011; Ehrlich, Ross, Chen, \& Miller, 2016; Miller, Cohen, \& Ritchey, 2002) and psychosis in adulthood (Khandaker, Pearson, Zammit, Lewis, \& Jones, 2014). A recent adult brain imaging study reported that an association between higher CRP levels and higher depressive symptoms was mediated by decreased functional connectivity between the ventromedial prefrontal cortex and the ventral striatum and the presupplementary motor cortex (Felger et al., 2016). Hence, animal and human research support the idea that adversity-related inflammation can shape neurodevelopment, and may also underlie behavioral and emotional disorders.

\section{The Potential Role of DNA Methylation in Adversity, Inflammation, and Mental Health}

How might exposure to adversity become biologically embedded in such a way as to systematically affect offspring inflammation and brain development? The developmental origins of health and disease hypothesis posits that pre- and postnatal environmental exposures can influence long-term vulnerability for physical and mental health problems (Wadhwa, Buss, Entringer, \& Swanson, 2009). One of the mechanistic drivers of these vulnerabilities is thought to be epigenetic alterations, such as DNA methylation (Gluckman et al., 2005). With regard to prenatal effects, few published studies exist that explicitly link prenatal adversity with DNA methylation specific to immune functioning and child mental health; however, mounting evidence suggests that prenatal adversity is associated with inflammatory response of cord blood monocytes of the neonate (Sureshchandra et al., 2017). More specifically, DNA methylation in cord blood has been associated with perinatal risks for immune-related disorders (such as asthma), including caesarean section (Schlinzig, Johansson, Gunnar, Ekström, \& Norman, 2009), elevated levels of maternal immune markers (DeVries, Stern, Wright, \& Vercelli, 2017), birth complications (Zhang et al., 2016), maternal cigarette smoking (van Otterdijk, Binder, \& Michels, in press), and diet (Lee et al., 2013). These data are consistent with a much larger group of studies showing that prenatal maternal depression, anxiety, nutrition, and toxin exposure (e.g., cigarette smoking) are associated with changes in DNA methylation in the cord blood of newborns (Barker, Cecil, et al., in press; Binder \& Michels, 2013; Cecil et al., in press; Oberlander et al., 2008; Richmond et al., 2015), which, in turn, can increase vulnerability for neurological (Monk et al., 2016) and mental health problems (Rijlaarsdam et al., 2017). 
Emerging literature also suggests that exposure to adversity after birth can continue to affect DNA methylation in the immune system. For example, individuals born with lower birth weight and shorter duration of breast feeding show higher concentrations of CRP in adulthood (McDade et al., 2014; Tzoulaki et al., 2008), and DNA methylation may be involved. Major psychosocial stressors, such as child neglect, maltreatment, housing quality, and poverty, associate with DNA methylation in immune system genes (Cecil et al., 2016; Essex et al., 2013). More specifically, certain studies have examined cell cultures and shown that adversity-related DNA methylation in immune system genes can associate with the expression of both elevated and reduced plasma inflammatory biomarkers (e.g., McDade et al., 2017). Furthermore, in a study of adults ( $~ 20$ years of age) who grew up in lowincome neighborhoods, greater exposure to trauma and witnessing of community violence associated with lower levels of DNA methylation around the $I L-6$ gene promoter and, in turn, a higher production of IL-6 in response to the Trier Social Stress test (Janusek, Tell, Gaylord-Harden, \& Mathews, 2017).

A recent study identified potential DNA methylation correlates of low-grade, chronic inflammation. Here, Ligthart et al. (2016) performed a epigenome-wide association of serum CRP in adults ( $\sim 11$ years of age). They reported 58 CpG sites (across 45 genes) that reliably replicated across a large European $(n=8,863)$ discovery sample and an African American $(n=4,111)$ replication sample. The researchers further characterized the molecular and clinical relevance of the $\mathrm{CpG}$ sites through examining associations with gene expression and clinical outcomes. However, Ligthart et al. (2016) did not examine associations with prenatal and postnatal adversity or with mental health outcomes, which is a main aim of the present study.

\section{The Present Study}

Using prospective data drawn from a large population-based sample, prenatal and postnatal adversity; DNA methylation at birth, age 7, and ages 15-17; cognitive function at age 9; and internalizing and externalizing mental health problems spanning childhood to adolescence, the current study had three overall research aims. First, we aimed to create and replicate an inflammation-related CRP epigenetic polygenic score (i-ePGS) biomarker of low-grade inflammation in a developmentally sensitive framework. Here we aimed to validate an i-ePGS biomarker by associating DNA methylation of relevant loci at age 15-17 with serum CRP at ages 15 and 17, the age when the DNA methylation resource and serum CRP samples overlapped. We then aimed to create i-ePGS at birth and age 7 to examine the degree to which i-ePGS correlated over time, thereby raising the possibility of a biomarker that could index chronic, low-grade inflammation early in the life course. Second, we aimed to examine if the i-ePGS biomarker associated with cognitive function and internalizing and externalizing symptoms, which would represent multifinality of outcome (Cicchetti \& Rogosch, 1996). More spe- cifically, we examined whether i-ePGS would negatively affect cognitive function, which, in turn, would then increase vulnerability for both internalizing and externalizing problems. The third overall aim was to examine pre- and postnatal adversity-related effects. With regard to prenatal effects, we tested if adversity in utero might indirectly associate with internalizing and externalizing problems, by way of affecting i-ePGS (at birth) and cognitive function. With regard to postnatal effects, we similarly tested if postnatal adversity might indirectly associate with internalizing and externalizing problems, by way of affecting i-ePGS during childhood (age 7) and cognitive function.

\section{Method}

\section{Participants}

Study participants were drawn from the Accessible Resource for Integrated Epigenomics Studies (ARIES; www.ariesepigenomics.org.uk; Relton et al., 2015), containing DNA methylation data for a subset of 1,018 mother-offspring pairs and nested within the Avon Longitudinal Study of Parents and Children (ALSPAC). ALSPAC is an ongoing epidemiological study of children born from 14,541 pregnant women residing in Avon, United Kingdom, with an expected delivery date between April 1991 and December 1992 (85\% of eligible population; Fraser et al., 2013). Informed consent was obtained from all ALSPAC participants, and ethical approval was obtained from the ALSPAC Law and Ethics Committee as well as local research committees. The original ALSPAC sample is representative of the general population (Boyd et al., 2013). Please note that the study website contains details of all the data that is available through a fully searchable data dictionary (http://www.bris.ac.uk/alspac/researchers/ dataaccess/data-dictionary/). For this study, we included youth from ARIES who had available data on symptomatology ratings (ages 7-15) as well as epigenetic data at birth $(n=$ $758,50 \%$ male). The cohort profile of ARIES by Relton et al. (2015) compared a selection of maternal characteristics in ARIES $(n=1,018)$ to the rest of the ALSPAC sample. ARIES versus ALSPAC mothers were more ethnically homogenous ( $\%$ white: ARIES $=100 \%$ vs. ALSPAC 97.4\%), slightly older (mean age: ARIES $=29.2$ vs. ALSPAC $=$ 28.2), less likely to have a manual occupation (ARIES = $14 \%$ vs. $\mathrm{ALSPAC}=20.5 \%$ ), and less likely to have smoked throughout pregnancy $($ ARIES $=9.7 \%$ vs. ALSPAC $=$ $19.4 \%$ ). Otherwise, the subsample was considered to be reasonably representative of the main ALSPAC population.

\section{Measures}

Child cognitive function was measured at age 8 by the Wechsler Intelligence Scale for Children (WISC-III UK; Weschsler, Golombok, \& Rust, 1992). The WISC-III UK was administered to the children at an ALSPAC research clinic. A short form of the measure was employed to reduce 
the likelihood that the children would become fatigued and that this would affect their performance on the WISC-III UK. The WISC-III UK comprises five verbal subtests (information, similarities, arithmetic, vocabulary, and comprehension) and five performance subtests (picture completion, coding, picture arrangement, block design, and object assembly). We extracted the common variance between the verbal and performance tests in a latent factor of cognitive function in the overall model. We did so as we sought to tap the true score (i.e., the common variance) of cognitive function, while accounting for potential measurement error in the verbal and performance tests, respectively (see Brown, 2014). Scores were age-normed in accordance with standard procedures. The distribution of verbal tests $(M=110.18, S D=16.52$, skew $=-0.002)$ and the performance tests $(M=101.81$, $S D=16.99$, skew $=-0.054$ ) were normal and within the range of age-appropriate norms.

Internalizing problems. We assessed internalizing problems via mother ratings at ages $7,10,13$, and 15 years, using the well-validated Development and Well-Being Assessment interview (DAWBA; Goodman, Heiervang, Collishaw, \& Goodman, 2011). The DAWBA was administered via a computer-based package of questionnaires, interviews, and rating techniques used to assess adolescent psychopathology based on DSM-IV criteria. Each question was introduced with the following stem: "Over the last 6 months, and as compared with other children the same age, has s/he often ..." followed by the specific clause. The response categories were $0=n o$ more than others, $1=$ a little more than others, and $2=a$ lot more than others. For depression, 12 symptoms were assessed, including "very sad," "lost interest," and "lost or gained weight." For generalized anxiety, 7 symptoms were assessed, including "worried about own behavior," "worried about health," and "worried about future." For both anxiety and depression, scales, per age, were summed and then used to create an overall latent variable that indexed common variance in internalizing problems across time.

Externalizing problems. Attention-deficit/hyperactivity disorder (ADHD), conduct disorder, and oppositional defiant disorder were also assessed via maternal ratings on the DAWBA at ages 7, 10, 13, and 15 years. ADHD comprised 18 symptoms, including "often fidgets," "blurted out answers before hearing questions properly," "does not listen," and "avoids things involving thought." For oppositional defiant disorder 9 symptoms were assessed, including "has temper outbursts," "has been touchy or easily annoyed," "argued with grown-ups," and "taken no notice of rules/refused to do as s/he is told." For conduct disorder 8 symptoms were assessed, including "used a weapon," "cruel to animals and birds," and "stealing on the streets." For all types of externalizing behaviors, scales, per age, were summed and then used to create an overall latent variable that indexed common variance in externalizing problems.
Adversity exposure. We assessed adversity exposure based on maternal reports. Risk items were organized into two developmental eras to reflect the timing of the i-ePGS (a) prenatal risks (18 weeks to 32 weeks) and (b) early childhood risks (birth-age 7). For each developmental period, items were organized to create distinct but correlated risk domains: (a) life events (e.g., death in family, accident, and illness), (b) contextual risks (e.g., poor housing conditions and financial problems), (c) parental risks (e.g., parental psychopathology, criminal involvement, and substance use), (d) interpersonal risks (e.g., intimate partner violence and family conflict), and (e) direct victimization (e.g., child bullied by peers or physically hurt; this measure of adversity exposure was specific to the postnatal risk composite).

These adversity scores were previously estimated and validated by Cecil et al. (2014), who used confirmatory factor analyses to assess the internal reliability of the individual risk domains and extract one adversity score for each developmental era. Model fit was good: (a) cumulative prenatal risk score: $\chi^{2}(2)=0.60, p=.73$; (b) cumulative early childhood risk score: $\chi^{2}(5)=14.94, p=.01$; comparative fit index $(\mathrm{CFI})=0.95$; Tucker-Lewis index $(\mathrm{TLI})=0.90$; root mean square error of approximation (RMSEA) $=0.07$; and $90 \%$ confidence interval $(\mathrm{CI})[0.03,0.12]$. In addition, we also incorporated, at the composite level, other risks pertinent to inflammation, including low birth weight $(<2500 \mathrm{~g})$ to the prenatal adversity, and breastfeeding $(1=$ no for first 6 months after birth, $0=$ yes in the first 6 months) and body mass index (at age 7) to the postnatal adversity index. Saved factor scores were used in all analyses.

DNA methylation data. A sample of $500 \mathrm{ng}$ genomic DNA from blood (cord at birth; whole blood at age 7 and age 15-17 years) was bisulfite-converted using the EZ-DNA methylation kit (Zymo Research, Orange, CA, USA). DNA methylation was quantified using the Illumina HumanMethylation450 BeadChip (HM450k; Illumina, USA) with arrays scanned using an Illumina iScan (software version 3.3.28). Samples or probes that failed quality control ( $>1 \%$ probes/ samples with background detection $p$ value $\geq .05$ ) were excluded from further analysis. Sex checks were performed using $\mathrm{X} / \mathrm{Y}$ chromosome methylation. Genotype probes on the HM450k were compared between samples from the same individual and against single nucleotide polymorphism (SNP)-chip data to identify and remove any sample mismatches. Samples were quantile normalized using the dasen function within the wateRmelon package (version 1.4.0) in R. Normalization performance was evaluated using all three testing metrics in wateRmelon (genki assessing SNP-related probes, dmrse assessing imprinted probes and seabi, assessing gender differences). Methylation levels were then indexed by beta values (corresponding to the ratio of the methylated signal divided by the sum of the methylated and unmethylated signal). Probes known to be cross-reactive or polymorphic (Chen et al., 2013; Price et al., 2013) and SNP (i.e., "rs") probes were removed. We also removed partici- 
pants with non-Caucasian or missing ethnicity (based on selfreports). Cell type proportions (CD8 T lymphocytes, CD4 T lymphocytes, natural killer cells, B lymphocytes, monocytes, and granulocytes) for each participant were estimated using the reference-based approach detailed in Houseman et al. (2012). These cell types were controlled for at each time point (i.e., birth, age 7, and age 15-17) in all analyses. In addition, for DNA methylation data at 15-17 years, we also controlled for age.

\section{Analyses}

Step 1: i-ePGS and validation. CRP-related probes were identified based on a recent epigenome-wide association study by Ligthart et al. (2016). We limited our selection to 7 probes (spanning a total of 9 genes) that showed the strongest evidence for a functional association with CRP levels (Table 1). Specifically, these probes were found to associate with (a) plasma CRP levels in both a discovery meta-analysis (9 cohorts, $n=8,863$ ) and a replication meta-analysis (4 cohorts, $n=4,111$ ); (b) whole-blood gene expression levels; and (c) at least one cardiometabolic phenotype of relevance to CRP (e.g., body mass index, coronary heart disease, lipids, etc.). We grouped the probes into a single i-ePGS. Specifically, we applied a method typically used for polygenic risk scores, which has recently been extended to epigenetic data (Shah et al., 2015), where we multiplied the ALSPAC methylation values by their respective standardized regression betas (i.e., weights) from the Ligthart et al. (2016) epigenome-wide association study, and then summed these weighted methylation values together into the i-ePGS. The use of weights ensured that the probes maintained their relative magnitude of association with CRP. We then validated the i-ePGS in ALSPAC by testing whether it significantly associated with measures of CRP at ages 15 and 17, as these are the ages where the i-ePGS and CRP overlap. Once the i-ePGS at age 15-17 was characterized and validated, we then created the same i-ePGS risk measure at earlier time points, using DNA methylation data at birth and age 7. We then examined bivariate correlations among the i-ePGS and between those scores and the measures of adversity, cognitive function, and psychopathology.

In this step we also examined, for each probe within the i-ePGS, possible genetic influences on the level of methylation. We did so by searching the mQTLdb database (http://www.mqtldb.org). The mQTLdb database contains the results of a large-scale study based on ARIES data, characterizing genome-wide significant SNP effects on DNAm levels for all Illumina 450k probes (see Gaunt et al., 2016).

Step 2: Associations between i-ePGS, cognitive function, and outcomes. Here we examined a latent path analysis examining the interrelations between i-ePGS, cognitive function, and child externalizing and internalizing symptoms. In this step, we also examined the degree to which i-ePGS at birth might indirectly relate to internalizing and/or externalizing symptoms via an i-ePGS at age 7 and/or cognitive function at age 7.

Indirect pathways were programmed in model constraint statements in Mplus (Muthén \& Muthén, 1998-2013). The indirect effects tested in this step assessed the extent to which i-ePGS might relate to higher internalizing/externalizing symptoms via cognitive function. Therefore, the indirect effects were defined by the product term of the pathways of interest (e.g., i-ePGS at birth to cognitive function BY cognitive function to internalizing symptoms). Because standard errors underlying indirect effects (i.e., product terms) are known to be skewed, we bootstrapped all indirect effects 5,000 times with bias corrected $95 \%$ CIs. The indirect pathways reported below are based on the bootstrapped variability around the product of nonstandardized path coefficient estimates.

Step 3: Associations between adversity, i-ePGS cognitive function, and outcomes. In this step, we tested the prenatal and postnatal effects by including the adversity scores (prenatal

Table 1. DNAm sites used in the inflammation-related epigenetic polygenic risk scores: Associations with CRP and gene expression from the original study (Ligthart et al.), with the addition of mQTLs

\begin{tabular}{|c|c|c|c|c|c|c|c|}
\hline \multirow[b]{2}{*}{ Marker name } & \multirow[b]{2}{*}{ Gene } & \multirow{2}{*}{$\begin{array}{l}\text { Methylation CRP } \\
\text { (discovery sample) }\end{array}$} & \multicolumn{2}{|c|}{$\begin{array}{l}\text { Methylation } \\
\text { expression }\end{array}$} & \multicolumn{2}{|c|}{$\begin{array}{c}\text { Expression CRP } \\
\text { levels }\end{array}$} & \multirow[b]{2}{*}{ mQTI } \\
\hline & & & $Z$ score & $p$ value & $Z$ score & $p$ value & \\
\hline \multirow[t]{2}{*}{ cg06126421 } & IER3 & -0.0052 & -3.98 & $6.90 \mathrm{E}-05$ & -1.39 & .16 & - \\
\hline & $T U B B$ & & -5.12 & $3.02 \mathrm{E}-07$ & -0.98 & .33 & - \\
\hline $\operatorname{cg06690548}$ & SLC7A11 & -0.0048 & -6.96 & $3.34 \mathrm{E}-12$ & 4.90 & $9.80 \mathrm{E}-07$ & - \\
\hline \multirow[t]{2}{*}{ cg10636246 } & AIM2 & -0.0069 & -12.37 & $3.89 \mathrm{E}-35$ & 11.01 & $3.40 \mathrm{E}-28$ & - \\
\hline & IFI16 & & -6.90 & $5.07 \mathrm{E}-12$ & 6.47 & $9.90 \mathrm{E}-11$ & - \\
\hline $\operatorname{cg} 18181703$ & SOCS3 & -0.0053 & -4.95 & $7.47 \mathrm{E}-07$ & 3.74 & $1.80 \mathrm{E}-04$ & - \\
\hline cg19821297 & DNASE2 & -0.0051 & -4.39 & $1.16 \mathrm{E}-05$ & 1.48 & .14 & Cis \\
\hline $\operatorname{cg} 25325512$ & $F G D 2$ & -0.0031 & -4.30 & $1.73 \mathrm{E}-05$ & -0.29 & .77 & - \\
\hline $\operatorname{cg} 27023597$ & HEATR6 & -0.0050 & -4.08 & $4.51 \mathrm{E}-05$ & 1.89 & .06 & Cis \\
\hline
\end{tabular}

Note: Bolded text represents CG probes that significantly associated with serum C-reactive protein (CRP) in adolescence within the ALSPAC cohort. 
and postnatal). This enabled us to trace the interrelationships between environmental exposures, the i-ePGS (birth and age 7), latent cognitive function (age 7), and the internalizing and externalizing (age 7-15) scores. For the prenatal effects, we expected i-ePGS at birth to associate with internalizing and/or externalizing scores indirectly via cognitive function at age 7 . For postnatal effects, postnatal adversity would associate with internalizing and/or externalizing problems indirectly via i-ePGS and cognitive function at age 7 . Indirect effects were estimated in identical fashion as Step 2.

Path analyses were conducted using Mplus version 7.11 (Muthén \& Muthén, 1998-2013). Model fit was determined through the CFI and TLI (acceptable fit $=>0.90$; Bentler $\&$ Bonett, 1980) and RMSEA (acceptable fit $=<0.08$; Browne \& Cudeck, 1993). Maximum likelihood estimation with robust standard errors was used to estimate the model parameters, and missing data were handled through full information maximum likelihood. All analyses were conducted using SAS v9.4 (ref) and Mplus v7.11 (Muthén \& Muthén, 1998-2016).

\section{Missing data}

For the 1,018 families within the ARIES resource, 914 had the required methylation data available from cord blood. Twenty-five samples at birth failed quality control $(>1 \%$ probes/samples with background detection $p$ value $\geq .05$ ) and were excluded from further analysis. From the resulting cord blood sample of 889 , participants with missing ethnicity were removed $(n=61)$. This resulted in a final total of 828 with cord blood samples at birth. We included participants with data for the CRP, DAWBA symptoms, risk factors, and DNA methylation. This resulted in a sample of 785 youth. We compared the 785 to the 828 on the study variables (i.e., prenatal risks and DAWBA symptoms) and found no significant differences.

\section{Results}

\section{Step 1: i-ePGS validation}

Table 1 contains the 7 probes from Ligthart et al. (2016) described above (see Supplemental Table 1, for total probe information). As can be seen, these probes were annotated to 9 total genes. For most genes, peripheral expression was higher than central nervous system expression; however, certain of these genes (e.g., $T U B B$ ) show moderate expression in the brain (see Supplemental Table 2). Two probes, cg19821297 and cg27023597, showed evidence of mQTL (i.e., genetic influences on DNA methylation). Associations between the i-ePGS at age 15-17 and CRP levels at ages 15 and 17 were examined, as this was the age and time span of overlapping DNAm and CRP. CRP associated with i-ePGS at age $15(r=-.111, p<.002)$ and age 17 $(r=-.181, p<.0001)$. We then created i-ePGS at earlier time points (i.e., birth and age 7). Participants' i-ePGS at age 15-17 autocorrelated with i-ePGS at age 7 ( $r=.314, p$ $<.0001)$ and at birth $(r=.175, p<.0001)$. To aid in the interpretation of environmental and phenotypic association with the i-ePGS, we took the inverse value of the i-ePGS. This transformation made the i-ePGS consistent with the direction of higher CRP for potential low-grade chronic inflammation. As can be seen in Table 2, i-ePGS at birth and age 7 differentially associated with the study variables.

\section{Step 2: Associations between i-ePGS, cognitive function, and outcomes}

Prior to describing the latent path model, we first highlight certain bivariate correlations between the mental health outcomes and the study variables (see Table 2). First, we found that both internalizing and externalizing symptoms associated negatively with cognitive function; internalizing (but not externalizing) problems associated with i-ePGS at age 7; and neither outcome associated with i-ePGS at birth. Second, cognitive function associated negatively with i-ePGS at birth, and the prenatal and postnatal adversity indexes. As stated earlier, i-ePGS at birth associated with a i-ePGS at age 7. We note also that cell type and sex each associated with i-ePGS (birth and age 7), and that sex associated with higher internalizing (females $>$ males) and externalizing symptoms (females < males) (age 7-15).

Figure 1 contains the latent path model that showed adequate fit to the data: $\chi^{2}(39)=77.395, p<.001 ; \mathrm{CFI}=$ $0.972 ;$ TLI $=0.954 ;$ RMSEA $=0.035,90 \%$ CI $[0.024$, 0.047]. Only significant pathways are shown, but full model estimates are available in online-only Supplemental Figure S.1. As can be seen, the associations described above largely remained significant after accounting for the other parameters in the model as well as cell type and sex. For example, i-ePGS at birth associated with both i-ePGS age 7 and lower cognitive function. In addition, i-ePGS at age 7 associated with higher internalizing, but not externalizing symptoms. Higher cognitive function associated with lower levels of both internalizing and externalizing symptoms. Moreover, indirect effects showed that i-ePGS at birth associated with higher internalizing, $b=0.020,95 \%$ CI [0.002, 0.069], and externalizing, $b=0.029,95 \%$ CI [0.003, 0.087], symptoms via lower cognitive function; and with higher internalizing symptoms, $b=0.032,95 \%$ CI [0.011, 0.064], but not externalizing symptoms, $b=0.005,95 \%$ CI [ $-0.011,0.029]$, via continuity of i-ePGS at age 7.

\section{Step 3: Associations between adversities, $i$-ePGS, cognitive function, and outcomes}

In this step we added the prenatal (18-32 weeks) and postnatal (birth-age 7) adversity scores and tested the prenatal and postnatal effects. Table 2 contains the correlations. At the bivariate level, pre- and postnatal adversity associated with internalizing and externalizing symptoms, and cognitive 


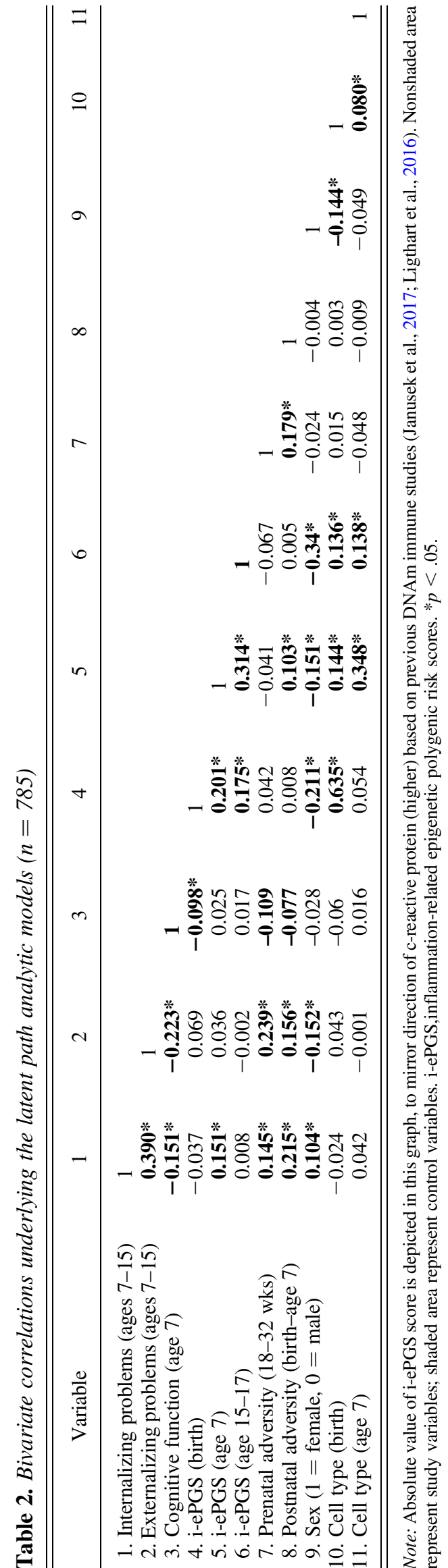

function. Postnatal, but not prenatal, adversity associated with i-ePGS at age 7.

Figure 2 contains the overall latent path model, which showed adequate fit to the data: $\chi^{2}(51)=96.122, p<$ .001 ; $\mathrm{CFI}=0.968$; $\mathrm{TLI}=0.947$; $\mathrm{RMSEA}=0.034,90 \%$ CI $[0.023,0.044]$. To decrease the complexity of Figure 2, only significant parameters are shown. Three results are highlighted here. First and foremost, as evidenced in the bivariate associations (and against our expectation), i-ePGS at birth did not associate with prenatal global adversity; we therefore have not included this variable in the model (see online-only Supplemental Figure S.2 for complete model estimates). Second, consistent with our expectation, postnatal adversity (birthage 7) associated with higher i-ePGS at age 7, which, in turn, associated with internalizing symptoms (age 7-15). Third, with the addition of adversity, two associations in the first model (Figure 1) changed due to the new conditional relationships. The association between higher i-ePGS at age 7 and lower cognitive function (age 7) increased in strength of association, and the association between higher cognitive function (age 7) and lower internalizing symptoms (age 7-15) decreased in strength of association.

On the one hand, these results suggest that the prenatal effects pathway does not involve the prenatal adversity index, but is confined to i-ePGS at birth, which might be a proxy for both unmeasured environmental effects during pregnancy and genetic influence (i.e., heritability) of immune system function. On the other hand, the data provides relatively straightforward support for postnatal effects, given that postnatal adversity associated with i-ePGS at age 7 . Three indirect effects were identified: i-ePGS at birth associated with lower cognitive function, which, in turn, associated with higher externalizing symptoms, $b=0.024 ; 95 \%$ CI [0.002, 0.078]; higher i-ePGS at age 7, which, in turn associated with higher internalizing symptoms, $b=0.030 ; 95 \%$ CI [0.003, 0.063]; and postnatal adversity (birth-age 7) associated with higher i-ePGS at age 7, which, in turn, associated with higher internalizing symptoms, $b=0.009 ; 95 \%$ CI [0.002, 0.022].

\section{Discussion}

In this prospective birth cohort study, we created and validated i-ePGS and then tested prenatal and postnatal hypotheses about child and adolescent mental health. There were two indirect effects stemming from i-ePGS at birth: one to higher externalizing problems ( $7-15$ years) via lower cognitive function (age 7), and the other to higher internalizing problems (7-15 years) via higher i-ePGS at age 7. These indirect pathways suggest multifinality, that a single risk factor can associate with multiple outcomes through different developmental pathways (Cicchetti \& Rogosch, 1996). We also identified one postnatal effect where adversity (birth to age 7) associated with higher internalizing problems (7-15 years) via higher i-ePGS at age 7. Our findings are consistent with previous research showing associations between early life adversity, inflammation-related DNAm, and vulnerability for 


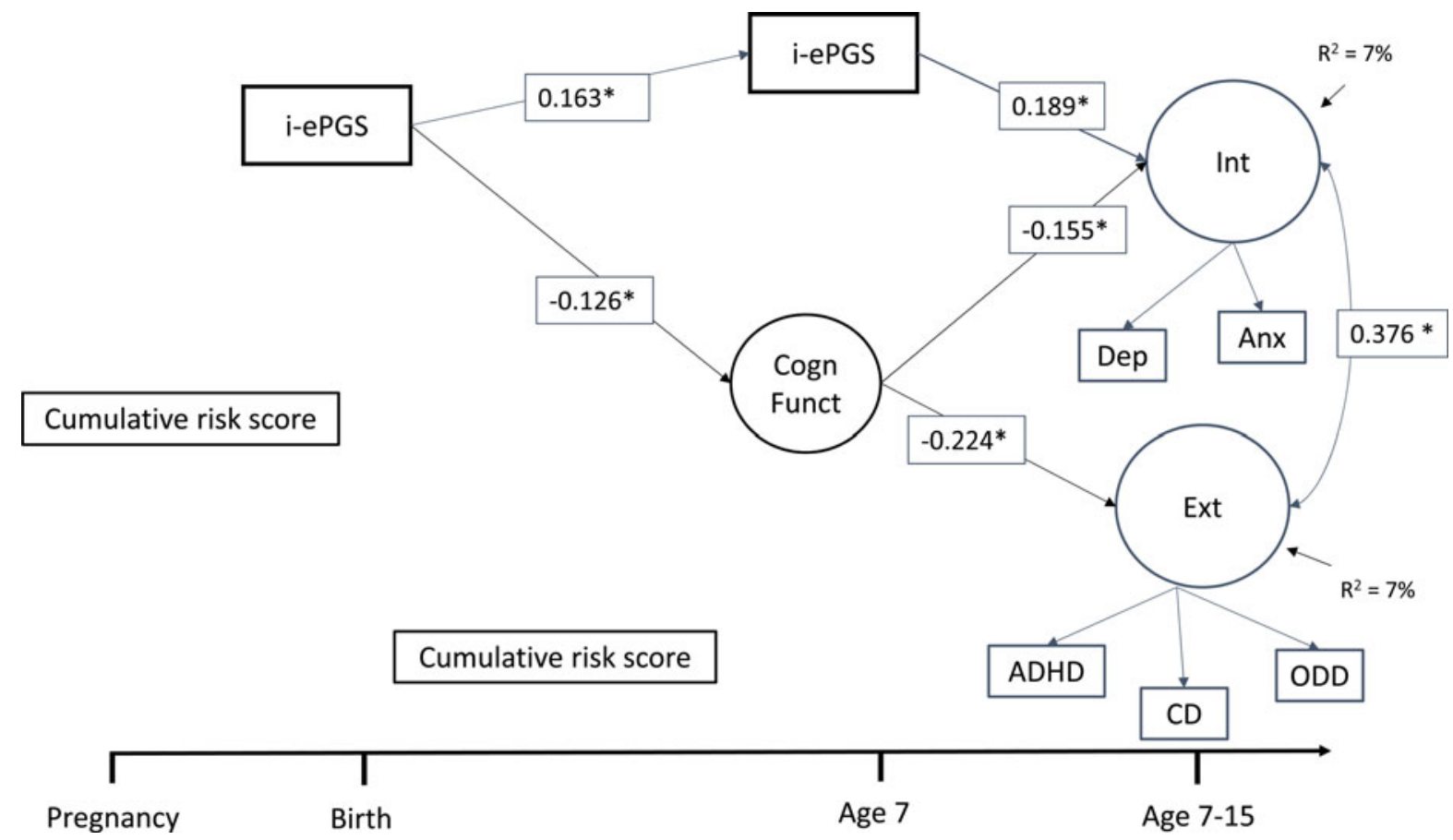

Figure 1. Latent path analytic model: Associations between i-ePGS, cognitive function, and internalizing and externalizing symptoms. ePGS, inflammation-related epigenetic risk score; inverse value of i-ePGS is depicted in this graph to mirror direction of C-reactive protein (higher) based on previous DNAm immune studies (Janusek et al., 2017; Ligthart et al., 2016) with only significant pathways shown, Int, internalizing symptoms. Ext, externalizing symptoms. Cogn Funct, cognitive function. Dep, depression symptoms. Anx, anxiety symptoms. ADHD, attention-deficit/hyperactivity disorder symptoms. CD, conduct disorder symptoms. ODD, oppositional defiant disorder symptoms.

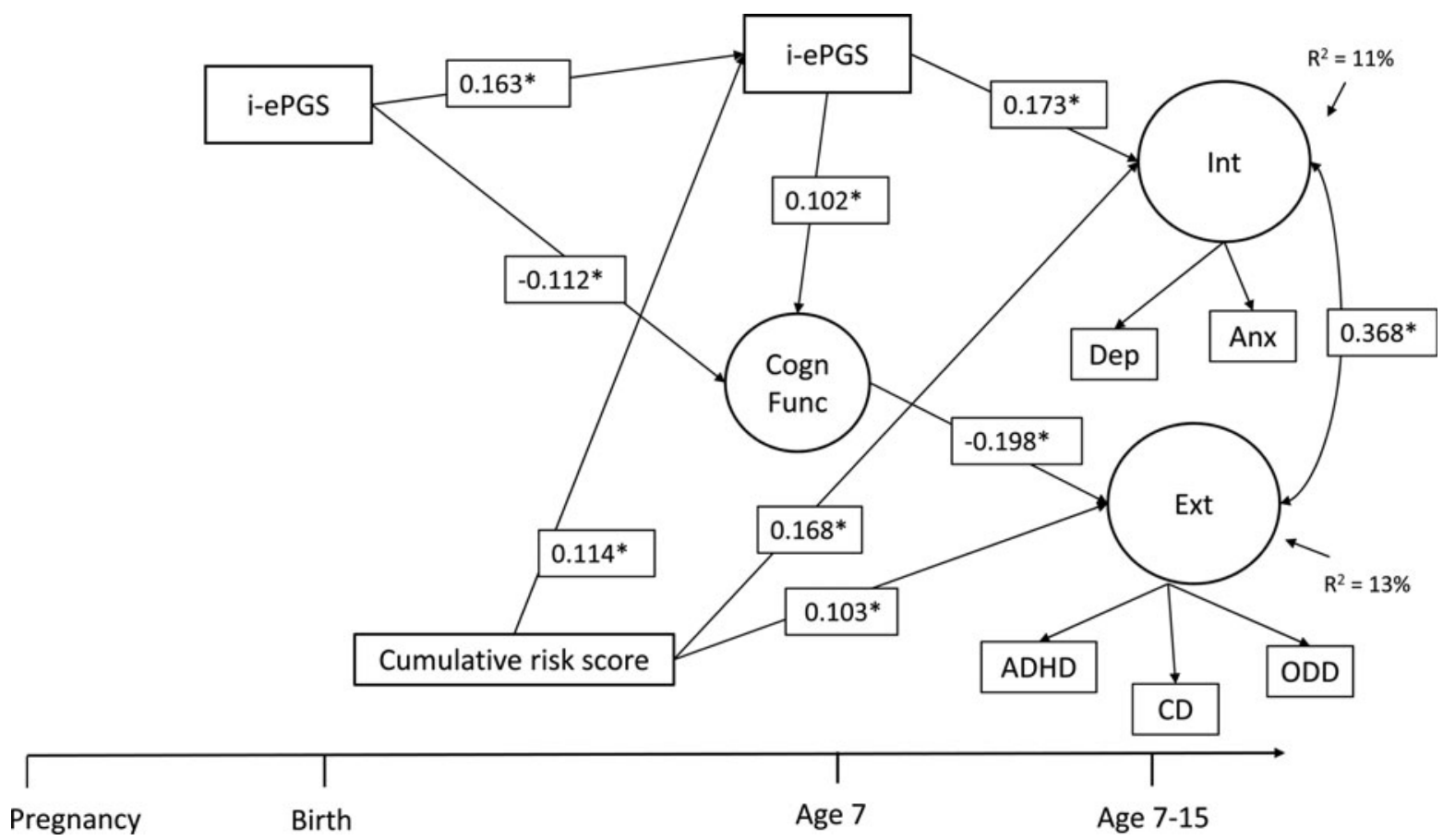

Figure 2. Latent path analytic model: Associations between cumulative risk score, i-ePGS, cognitive function, and internalizing and externalizing symptoms. i-ePGS, inflammation-related epigenetic risk score; inverse value of i-ePGS is depicted in this graph to mirror direction of Creactive protein (higher) based on previous DNAm immune studies (Janusek et al., 2017; Ligthart et al., 2016) with only significant paths shown. $* p<.05$. Int, internalizing symptoms. Ext, externalizing symptoms. Cogn Funct, cognitive function. Dep, depression symptoms. Anx, anxiety symptoms. ADHD, attention-deficit/hyperactivity disorder symptoms. CD, conduct disorder symptoms. ODD, oppositional defiant disorder symptoms. 
mental health (Janusek et al., 2017; McDade et al., 2014). The present findings expand this literature in three main ways.

First, as mentioned, we were interested in the potential role of immune system-related DNA methylation in influencing cognitive function and mental health. We focused on CRP, as it is an acute-phase protein marker of systemic inflammation produced in response to increasing levels of circulating inflammatory factors (Mitchell \& Aneshensel, 2017). In addition, serum CRP-related DNA methylation has recently been reported in an epigenome-wide association study by Ligthart et al. (2016), which was based on multiple adult cohorts. Based on these results, we created an i-ePGS and (partially) replicated Ligthart et al. (2016) study: the i-ePGS associated with overlapping measures of serum CRP at age 15-17. Taking this as acceptable validation, we then created i-ePGS at birth and age 7 .

Our second main finding was in the longitudinal associations between the i-ePGS and study variables. In the developmental origins of health and disease hypothesis, DNA methylation is suggested as a biological mechanism that can establish early vulnerability for subsequent mental and physical health problems (Monk et al., 2016). Here, for the first time, we show a new candidate mechanism: i-ePGS. Specifically, we found that i-ePGS at birth associated with lower cognitive function, which, in turn, associated with higher externalizing problems. This indirect effect is noteworthy as most research has reported direct associations between peripheral inflammation markers and internalizing, but not externalizing, problems (Danese \& Baldwin, 2017). However, it is worth noting that a recent epigenome-wide association study reported variation in DNA methylation in cytokine-related genes in adults with a history of chronic childhood physical aggression (Provençal et al., 2014). The neuroimmune network hypothesis (Nusslock \& Miller, 2016) posits that peripheral inflammation can associate with externalizing problems by way of affecting executive control-related processes linked to the prefrontal cortex, such as executive control, decision making, and regulating threat- and reward-related tendencies (see Danese \& Lewis, 2016; Hostinar, Nusslock, \& Miller, 2017). Of interest, our measure of cognitive function (i.e., the WISC-III) was composed of verbal subtests (e.g., vocabulary, comprehension, etc.) and performance subtests (e.g., block design, object assembly, etc.). These aspects of cognitive function have been associated with both infection/immune functioning (Jensen, Berens, \& Nelson, 2017) and externalizing behaviors (Barker et al., 2007; Loeber et al., 2012). Hence, the present results highlight the potential of inflammation associating with externalizing problems via cognitive function.

The age 7 i-ePGS directly associated with internalizing problems, which, as stated, supports a range of research that has shown inflammation to associate with depressive symptoms, anxiety, and posttraumatic stress disorder (Danese \& Baldwin, 2017). The differences between this present study and those former studies are that (a) the association was not contingent on individuals being clustered by exposure to ad- versity (e.g., see Danese et al., 2011; Miller et al., 2002); and (b) we examined an i-ePGS biomarker that is associated with CRP. Why would i-ePGS associate with higher internalizing problems, or lower cognitive function, for that matter? Although highly speculative, the genetic annotation of the different CpGs in the i-ePGS may help in interpreting these associations. For example, $\operatorname{cg} 06126421$ is annotated to $T U B B$, a complex locus involved in the structural component of microtubules, mutations of which are reported to associate with aberrant neural development (Breuss et al., 2012). Of interest, this gene is expressed not only in peripheral samples but also in the brain, bone marrow, lymphocytes, and placental tissue (Fagerberg et al., 2014). TUBB may therefore be a gene involved in both prenatal and postnatal inflammatory effects on neural development and brain-based mental health problems. These associations, as well as the i-ePGS themselves, should be considered preliminary and in need of replication.

The third main finding is regarding the prenatal and postnatal adversity-related hypotheses. Against expectation, prenatal adversity did not associate with the i-ePGS at birth. On the one hand, this null result may indicate that we did not assess the correct types of immunity-related prenatal adversities (e.g., maternal obesity) or that CRP-related methylation changes do not capture the influence of prenatal adversities on the immune system. There are many other immune markers that could be examined. For example, interleukin-8, which can coincide or precede an increase in CRP, is shown to have specific $\mathrm{T}$ cell effector (i.e., pro-inflammatory) functions in neonates but not adults (Gibbons et al., 2014). On the other hand, i-ePGS at birth may have a strong heritable component above and beyond environmental influence. CRP is estimated to be moderately heritable (40\%; Baldwin et al., 2017; Pankow et al., 2001), and latent genetic risk can explain up to $15 \%$ of serum CRP variability (Baldwin et al., 2017). Moreover, a large-scale genome-wide association study (Dehghan et al., 2011) developed a weighted genetic risk score (similar to the weighted i-ePGS examined here) that was strongly associated with CRP levels. Another genome-wide association study identified genetic pathways related to immune system functioning for several types of mental health disorders (Network and Pathway Analysis Subgroup of the Psychiatric Genomics Consortium, 2015). Although we identified genetic influence (i.e., mQTL) on DNAm levels in one of the CpGs included in the i-ePGS, for this type of analysis to be more informative, we would need to also show that the SNP (rs1 1667764) is influential in cognitive function or internalizing symptoms, which we were unpowered to do. However, rs11667764 associates with variation in putamen and thalamus volume (Hibar et al., 2015), so it may be a SNP that is worthwhile of further examination.

It is of great interest that the postnatal global adversity index (birth-age 7) associated with i-ePGS at age 7, which in turn, associated with internalizing problems (age 7-15). This finding supports the "cumulative" adversity hypothesis (see Danese \& Baldwin, 2017), where repeated exposures of stressful events can become embedded in the epigenetic 
functioning of the immune system (Janusek et al., 2017), which can eventually manifest as chronic, low-grade inflammation (McDade et al., 2017) and vulnerability for mental health problems. However, also noteworthy is that this pathway did not include the measure of cognitive function. A recent meta-analysis has reported that the type of measure employed here may index impaired cognitive function that is concurrent to, but not predictive of, internalizing symptoms (see Scult et al., 2017), which brings up the possibility that a different measure of brain function may be more appropriate. The neuroimmune network hypothesis, for example, highlights sensitivity to rewards and reward-related brain function in networked corticobasal ganglia regions, as the most highly relevant for internalizing problems (Hostinar et al., 2017). Future research may want to examine dampened reward sensitivity as a risk for future internalizing problems.

Findings should be interpreted in light of a number of limitations. First, the current study was based on a modestly sized population-based sample of youth. In the future, it will be important to test the robustness of findings using other epidemiological cohorts. Second, the study focused exclusively on i-ePGS as a proxy for CRP; additional research is needed to establish that these i-ePGS are developmentally sensitive, by way of examining CRP associations at each age. Third,

\section{References}

Andersson, N. W., Li, Q., Mills, C. W., Ly, J., Nomura, Y., \& Chen, J. (2016). Influence of prenatal maternal stress on umbilical cord blood cytokine levels. Archives of Women's Mental Health, 19, 761-767.

Babri, S., Doosti, M.-H., \& Salari, A.-A. (2014). Strain-dependent effects of prenatal maternal immune activation on anxiety- and depression-like behaviors in offspring. Brain, Behavior, and Immunity, 37, 164-176.

Baldwin, J. R., Arseneault, L., Caspi, A., Fisher, H. L., Moffitt, T. E., Odgers, C. L., . . Kepa, A. (2017). Childhood victimization and inflammation in young adulthood: A genetically sensitive cohort study. Brain, Behavior, and Immunity. Advance online publication.

Barker, E. D., Cecil, C. A. M., Walton, E., Jaffee, S. R., Rowe, R., Maughan, B., ... Gaunt, T. R. (in press). A methylome-wide association study of trajectories of oppositional defiant behaviors and biological overlap with attention deficit hyperactivity disorder. Child Development. doi:10.1111/ cdev.12957

Barker, E. D., Kirkham, K., Ng, J., \& Jensen, S. M. K. (2013). Prenatal maternal depression, prenatal nutrion and child congitive function. British Journal of Psychiatry, 203, 417-421.

Barker, E. D., Seguin, J. R., White, H. R., Bates, M. E., Lacourse, E., Carbonneau, R., \& Tremblay, R. E. (2007). Developmental trajectories of male physical violence and theft: Relations to neurocognitive performance. Archives of General Psychiatry, 64, 592-599. doi:10.1001/archpsyc.64.5.592

Barker, E. D., Walton, E., \& Cecil, C. A. M. (in press). Annual Research Review: DNA methylation as a mediator in the association between risk exposure and child and adolescent psychopathology. Journal of Child Psychology and Psychiatry. doi:10.1111/jcpp.12782

Bentler, P. M., \& Bonett, D. G. (1980). Significance tests and goodness of fit in the analysis of covariance structures. Psychological Bulletin, 88, 588-606.

Binder, A. M., \& Michels, K. B. (2013). The causal effect of red blood cell folate on genome-wide methylation in cord blood: A Mendelian randomization approach. BMC Bioinformatics, 14, 1.

Boyd, A., Golding, J., Macleod, J., Lawlor, D. A., Fraser, A., Henderson, J., . . . Davey Smith, G. (2013). Cohort Profile: The "Children of the 90s"-The index offspring of the Avon Longitudinal Study of Parents and Children. International Journal of Epidemiology, 42, 111-127. doi:10.1093/ije/dys064

Breuss, M., Heng, J. I.-T., Poirier, K., Tian, G., Jaglin, X. H., Qu, Z., . . . Haas, M. (2012). Mutations in the $\beta$-tubulin gene TUBB5 cause microcephaly with structural brain abnormalities. Cell Reports, 2, 1554-1562. findings were based on a certain measure of cognitive function (i.e., WISC-III) that has previously been associated with both internalizing and externalizing problems. Therefore, research will be needed to establish the relevance of the present findings to both brain structure and function, as well as other measures of cognitive function. Fourth, despite the fact that we identified prospective associations between iePGS and internalizing and externalizing problems, it is not possible to establish causality, as associations could reflect the contribution of confounding genetic and environmental influences. Fifth, adversity can associate with inflammatory response through bidirectional interactions between the immune system and the neuroendocrine stress axes. Animal research has shown that, for instance, prolonged stress can lead to an attenuation of the anti-inflammatory properties of glucocorticoid and contribute to chronic low-grade inflammation (Miller, Chen, \& Parker, 2011). It will be important for future research to examine how the neuroendocrine stress system might further inform the present results.

\section{Supplementary Material}

To view the supplementary material for this article, please visit https://doi.org/10.1017/S0954579418000330.

Brown, T. A. (2014). Confirmatory factor analysis for applied research. New York: Guilford Press.

Browne, M. W., \& Cudeck, R. (1993). Alternative ways of assessing model fit. In K. A. Bollen \& J. S. Long (Eds.), Testing structural equation models (pp. 136-162). Newburry Park, CA: Sage.

Cecil, C. A. M., Jaffee, S. R., O'Connor, T. G., Oullet-Morin, I., Smith, R., Relton, C. L., . . Barker, E. D. (in press). Neonatal DNA methylation and early-onset conduct problems: A genome-wide, prospective study. Development and Psychopathology.

Cecil, C. A. M., Lysenko, L., Jaffee, S. R., Relton, C. L., Mill, J., \& Barker, E. D. (2014). Environmental risk, oxytocin receptor gene (OXTR) methylation and youth callous-unemotional traits: A 13-year longitudinal study. Molecular Psychiatry, 19, 1071-1077. doi:10.1038/mp.2014.95

Cecil, C. A. M., Smith, R. G., Walton, E., Mill, J., McCrory, E. J., \& Viding, E. (2016). Epigenetic signatures of childhood abuse and neglect: Implications for psychiatric vulnerability assessment. Journal of Psychiatric Research, 83, 184-194. doi:10.1016/j.jpsychires.2016.09.010

Chen, Y.-A., Lemire, M., Choufani, S., Butcher, D. T., Grafodatskaya, D., Zanke, B. W., . . . Weksberg, R. (2013). Discovery of cross-reactive probes and polymorphic CpGs in the Illumina Infinium HumanMethylation450 microarray. Epigenetics, 8, 203-209.

Cicchetti, D., \& Rogosch, F. (1996). Equifinality and multifinality in developmental psychopathology. Development and Psychopathology, 8, 597-600.

Coussons-Read, M. E., Okun, M. L., \& Nettles, C. D. (2007). Psychosocial stress increases inflammatory markers and alters cytokine production across pregnancy. Brain, Behavior, and Immunity, 21, 343-350.

Danese, A., \& Baldwin, J. R. (2017). Hidden wounds? Inflammatory links between childhood trauma and psychopathology. Annual Review of Psychology, 68, 517-544.

Danese, A., Caspi, A., Williams, B., Ambler, A., Sugden, K., Mika, J., . . Moffitt, T. (2011). Biological embedding of stress through inflammation processes in childhood. Molecular Psychiatry, 16, 244.

Danese, A., \& Lewis, S. J. (2016). Psychoneuroimmunology of early-life stress: The hidden wounds of childhood trauma? Neuropsychopharmacology. Advance online publication.

Danese, A., Moffitt, T. E., Harrington, H., Milne, B. J., Polanczyk, G., Pariante, C. M., . . . Caspi, A. (2009). Adverse childhood experiences and adult risk factors for age-related disease: Depression, inflammation, 
and clustering of metabolic risk markers. Archives of Pediatrics and Adolescent Medicine, 163, 1135-1143.

Dantzer, R., O'Connor, J. C., Freund, G. G., Johnson, R. W., \& Kelley, K. W. (2008). From inflammation to sickness and depression: When the immune system subjugates the brain. Nature Reviews Neuroscience, 9, 46.

Dehghan, A., Dupuis, J., Barbalic, M., Bis, J. C., Eiriksdottir, G., Lu, C., . . . Henneman, P. (2011). Meta-analysis of genome-wide association studies in $>80000$ subjects identifies multiple loci for C-reactive protein levels. Circulation, 123, 731-738.

DeVries, A., Stern, D. A., Wright, A. L., \& Vercelli, D. (2017). Neonatal DNA methylation profiles are associated with the maternal prenatal immune milieu selectively in children with non-asthmatic mothers. Paper presented at the American Thoracic Society 2017 International Conference, Washington, DC, May 19-24, 2017.

Ehrlich, K. B., Ross, K. M., Chen, E., \& Miller, G. E. (2016). Testing the biological embedding hypothesis: Is early life adversity associated with a later proinflammatory phenotype? Development and Psychopathology, $28(4$, pt. 2), 1273-1283.

Essex, M. J., Thomas Boyce, W., Hertzman, C., Lam, L. L., Armstrong, J. M., Neumann, S. M. A., \& Kobor, M. S. (2013). Epigenetic vestiges of early developmental adversity: Childhood stress exposure and DNA methylation in adolescence. Child Development, 84, 58-75. doi:10. 1111/j.1467-8624.2011.01641.x

Fagerberg, L., Hallström, B. M., Oksvold, P., Kampf, C., Djureinovic, D., Odeberg, J., . . Edlund, K. (2014). Analysis of the human tissue-specific expression by genome-wide integration of transcriptomics and antibodybased proteomics. Molecular \& Cellular Proteomics, 13, 397-406.

Felger, J. C., Li, Z., Haroon, E., Woolwine, B. J., Jung, M. Y., Hu, X., \& Miller, A. H. (2016). Inflammation is associated with decreased functional connectivity within corticostriatal reward circuitry in depression. Molecular Psychiatry, 21, 1358.

Frank, M. G., Hershman, S. A., Weber, M. D., Watkins, L. R., \& Maier, S. F. (2014). Chronic exposure to exogenous glucocorticoids primes microglia to pro-inflammatory stimuli and induces NLRP3 mRNA in the hippocampus. Psychoneuroendocrinology, 40, 191-200.

Fraser, A., Macdonald-Wallis, C., Tilling, K., Boyd, A., Golding, J., Davey Smith, G., . . Lawlor, D. A. (2013). Cohort Profile: The Avon Longitudinal Study of Parents and Children: ALSPAC mothers cohort. International Journal of Epidemiology, 42, 97-110. doi:10.1093/ije/dys066

Gaunt, T. R., Shihab, H. A., Hemani, G., Min, J. L., Woodward, G., Lyttleton, O., . . . Ring, S. M. (2016). Systematic identification of genetic influences on methylation across the human life course. Genome Biology, 17, 61.

Gibbons, D., Fleming, P., Virasami, A., Michel, M.-L., Sebire, N. J., Costeloe, K., . . Hayday, A. (2014). Interleukin-8 (CXCL8) production is a signatory $\mathrm{T}$ cell effector function of human newborn infants. Nature Medicine, 20, 1206-1210.

Gluckman, P. D., Hanson, M. A., Spencer, H. G., \& Bateson, P. (2005). Environmental influences during development and their later consequences for health and disease: Implications for the interpretation of empirical studies. Proceedings of the Royal Society of London B: Biological Sciences, 272, 671-677.

Goasdoué, K., Miller, S. M., Colditz, P. B., \& Bjorkman, S. T. (2016). The blood-brain barrier: Protecting the developing fetal brain. Placenta. Advance online publication.

Goodman, A., Heiervang, E., Collishaw, S., \& Goodman, R. (2011). The "DAWBA bands" as an ordered-categorical measure of child mental health: Description and validation in British and Norwegian samples. Social Psychiatry and Psychiatric Epidemiology, 46, 521-532. doi:10. 1007/s00127-010-0219-x

Hibar, D. P., Stein, J. L., Renteria, M. E., Arias-Vasquez, A., Desrivières, S., Jahanshad, N., ... Andersson, M. (2015). Common genetic variants influence human subcortical brain structures. Nature, 520, 224-229.

Hostinar, C. E., Nusslock, R., \& Miller, G. E. (2017). Future directions in the study of early-life stress and physical and emotional health: Implications of the neuroimmune network hypothesis. Journal of Clinical Child and Adolescent Psychology. Advance online publication.

Houseman, E. A., Accomando, W. P., Koestler, D. C., Christensen, B. C., Marsit, C. J., Nelson, H. H., . . Kelsey, K. T. (2012). DNA methylation arrays as surrogate measures of cell mixture distribution. BMC Bioinformatics, 13,1 .

Janusek, L. W., Tell, D., Gaylord-Harden, N., \& Mathews, H. L. (2017). Relationship of childhood adversity and neighborhood violence to a proinflammatory phenotype in emerging adult African American men: An epigenetic link. Brain, Behavior, and Immunity, 60, 126-135.
Jensen, S. K., Berens, A. E., \& Nelson, C. A., III. (2017). Effects of poverty on interacting biological systems underlying child development. Lancet Child \& Adolescent Health. Advance online publication.

Jensen, S. K., Dumontheil, I., \& Barker, E. D. (2014). Developmental interrelations between early maternal depression, contextual risks, and interpersonal stress, and their effect on later child cognitive functioning. $D e-$ pression and Anxiety, 31, 599-607.

Jiang, N. M., Tofail, F., Ma, J. Z., Haque, R., Kirkpatrick, B., Nelson., C. A., III, \& Petri, W. A., Jr. (2017). Early life inflammation and neurodevelopmental outcome in Bangladeshi infants growing up in adversity. American Journal of Tropical Medicine and Hygiene, 97, 974-979.

Joram, N., Boscher, C., Denizot, S., Loubersac, V., Winer, N., Roze, J., \& Gras-Le Guen, C. (2006). Umbilical cord blood procalcitonin and C reactive protein concentrations as markers for early diagnosis of very early onset neonatal infection. Archives of Disease in Childhood-Fetal and Neonatal Edition, 91, F65-F66.

Kaukola, T., Herva, R., Perhomaa, M., Pääkkö, E., Kingsmore, S., Vainionpää, L., \& Hallman, M. (2006). Population cohort associating chorioamnionitis, cord inflammatory cytokines and neurologic outcome in very preterm, extremely low birth weight infants. Pediatric Research, 59, 478-483.

Khandaker, G. M., Pearson, R. M., Zammit, S., Lewis, G., \& Jones, P. B (2014). Association of serum interleukin 6 and C-reactive protein in childhood with depression and psychosis in young adult life: A population-based longitudinal study. JAMA Psychiatry, 71, 1121-1128.

Knuesel, I., Chicha, L., Britschgi, M., Schobel, S. A., Bodmer, M., Hellings, J. A., . . Prinssen, E. P. (2014). Maternal immune activation and abnormal brain development across CNS disorders. Nature Reviews Neurology, 10, 643-660.

Lee, H.-S., Barraza-Villarreal, A., Hernandez-Vargas, H., Sly, P. D., Biessy, C., Ramakrishnan, U., . . . Herceg, Z. (2013). Modulation of DNA methylation states and infant immune system by dietary supplementation with $\omega-3$ PUFA during pregnancy in an intervention study. American Journal of Clinical Nutrition, 98, 480-487.

Ligthart, S., Marzi, C., Aslibekyan, S., Mendelson, M. M., Conneely, K. N., Tanaka, T., ... Guan, W. (2016). DNA methylation signatures of chronic low-grade inflammation are associated with complex diseases. Genome Biology, 17, 255.

Loeber, R., Menting, B., Lynam, D. R., Moffitt, T. E., Stouthamer-Loeber, M., Stallings, R., . . P Pardini, D. (2012). Findings from the Pittsburgh Youth Study: Cognitive impulsivity and intelligence as predictors of the age-crime curve. Journal of the American Academy of Child \& Adolescent Psychiatry, 51, 1136-1149.

McDade, T. W., Metzger, M. W., Chyu, L., Duncan, G. J., Garfield, C., \& Adam, E. K. (2014). Long-term effects of birth weight and breastfeeding duration on inflammation in early adulthood. Proceedings of the Royal Society Part B: Biological Sciences, 281, 20133116.

McDade, T. W., Ryan, C., Jones, M. J., MacIsaac, J. L., Morin, A. M., Meyer, J. M., ... Kuzawa, C. W. (2017). Social and physical environments early in development predict DNA methylation of inflammatory genes in young adulthood. Proceedings of the National Academy of Sciences, $114,7611-7616$

Miller, G. E., \& Chen, E. (2013). The biological residue of childhood poverty. Child Development Perspectives, 7, 67-73.

Miller, G. E., Chen, E., \& Parker, K. J. (2011). Psychological stress in childhood and susceptibility to the chronic diseases of aging: Moving toward a model of behavioral and biological mechanisms. Psychological Bulletin, 137, 959.

Miller, G. E., Cohen, S., \& Ritchey, A. K. (2002). Chronic psychological stress and the regulation of pro-inflammatory cytokines: A glucocorticoid-resistance model. Health Psychology, 21, 531.

Mitchell, U. A., \& Aneshensel, C. S. (2017). Social inequalities in inflammation Age variations in older persons. Journal of Aging and Health, 29, 769-787.

Monk, C., Feng, T., Lee, S., Krupska, I., Champagne, F. A., \& Tycko, B. (2016). Distress during pregnancy: Epigenetic regulation of placenta glucocorticoid-related genes and fetal neurobehavior. American Journal of Psychiatry, 173, 705-713.

Muthén, L. K., \& Muthén, B. O. (1998-2013). Mplus: Statistical analyses with latent variables. User's guide (6th ed.). Los Angeles: Author.

Muthén, L. K., \& Muthén, B. O. (1998-2016). Mplus: The comprehensive modeling program for applied researchers. User's guide (Version 7.22). Los Angeles: Author.

Network and Pathway Analysis Subgroup of the Psychiatric Genomics Consortium. (2015). Psychiatric genome-wide association study analyses implicate neuronal, immune and histone pathways. Nature Neuroscience, 18, 199-209. 
Nusslock, R., \& Miller, G. E. (2016). Early-life adversity and physical and emotional health across the lifespan: A neuroimmune network hypothesis. Biological Psychiatry, 80, 23-32.

Oberlander, T. F., Weinberg, J., Papsdorf, M., Grunau, R., Misri, S., \& Devlin, A. M. (2008). Prenatal exposure to maternal depression, neonatal methylation of human glucocorticoid receptor gene (NR3C1) and infant cortisol stress responses. Epigenetics, 3, 97-106.

O’Connor, T. G., Moynihan, J. A., \& Caserta, M. T. (2014). Annual Research Review: The neuroinflammation hypothesis for stress and psychopathology in children-Developmental psychoneuroimmunology. Journal of Child Psychology and Psychiatry, 55, 615-631.

Pankow, J. S., Folsom, A. R., Cushman, M., Borecki, I. B., Hopkins, P. N., Eckfeldt, J. H., \& Tracy, R. P. (2001). Familial and genetic determinants of systemic markers of inflammation: The NHLBI family heart study. Atherosclerosis, 154, 681-689.

Patrick, P. D., Oriá, R. B., Madhavan, V., Pinkerton, R. C., Lorntz, B., Lima, A. A., \& Guerrant, R. L. (2005). Limitations in verbal fluency following heavy burdens of early childhood diarrhea in Brazilian shantytown children. Child Neuropsychology, 11, 233-244.

Price, E. M., Cotton, A. M., Lam, L. L., Farré, P., Emberly, E., Brown, C. J., ... Kobor, M. S. (2013). Additional annotation enhances potential for biologically-relevant analysis of the Illumina Infinium HumanMethylation450 BeadChip array. Epigenetics \& Chromatin, 6, 1.

Provençal, N., Suderman, M. J., Guillemin, C., Vitaro, F., Côté, S. M., Hallett, M., ... Szyf, M. (2014). Association of childhood chronic physical aggression with a DNA methylation signature in adult human T cells. PLOS ONE, 9, e89839.

Relton, C. L., Gaunt, T., McArdle, W., Ho, K., Duggirala, A., Shihab, H., . . . Reik, W. (2015). Data resource profile: accessible resource for integrated epigenomic studies (ARIES). International Journal of Epidemiology, 44, 1181-1190.

Richmond, R. C., Simpkin, A. J., Woodward, G., Gaunt, T. R., Lyttleton, O., McArdle, W. L., . . . Tilling, K. (2015). Prenatal exposure to maternal smoking and offspring DNA methylation across the lifecourse: Findings from the Avon Longitudinal Study of Parents and Children (ALSPAC). Human Molecular Genetics, 24, 2201-2217.

Rijlaarsdam, J., Cecil, C. A., Walton, E., Mesirow, M. S., Relton, C. L., Gaunt, T. R., . . Barker, E. D. (2017). Prenatal unhealthy diet, insulinlike growth factor 2 gene (IGF2) methylation, and attention deficit hyperactivity disorder symptoms in youth with early-onset conduct problems. Journal of Child Psychology and Psychiatry, 58, 19-27.

Rock, P., Roiser, J., Riedel, W., \& Blackwell, A. (2014). Cognitive impairment in depression: A systematic review and meta-analysis. Psychological Medicine, 44, 2029-2040.

Schlinzig, T., Johansson, S., Gunnar, A., Ekström, T., \& Norman, M. (2009). Epigenetic modulation at birth-altered DNA-methylation in white blood cells after Caesarean section. Acta Paediatrica, 98, 1096-1099.
Scult, M. A., Paulli, A. R., Mazure, E. S., Moffitt, T. E., Hariri, A. R., \& Strauman, T. J. (2017). The association between cognitive function and subsequent depression: A systematic review and meta-analysis. Psychological Medicine, 47, 1-17.

Séguin, J. R., Nagin, D. S., Assaad, J.-M., \& Tremblay, R. E. (2004). Cognitive-neuropsychological function in chronic physical aggression and hyperactivity. Journal of Abnormal Psychology, 113, 603-613.

Shah, S., Bonder, M. J., Marioni, R. E., Zhu, Z., McRae, A. F., Zhernakova, A., ... Mendelson, M. M. (2015). Improving phenotypic prediction by combining genetic and epigenetic associations. American Journal of Human Genetics, 97, 75-85.

Sureshchandra, S., Wilson, R. M., Rais, M., Marshall, N. E., Purnell, J. Q., Thornburg, K. L., \& Messaoudi, I. (2017). Maternal pregravid obesity remodels the DNA methylation landscape of cord blood monocytes disrupting their inflammatory program. Journal of Immunology. Advance online publication.

Tilley, S. K., Joseph, R. M., Kuban, K. C., Dammann, O. U., O’Shea, T. M., \& Fry, R. C. (2017). Genomic biomarkers of prenatal intrauterine inflammation in umbilical cord tissue predict later life neurological outcomes. PLOS ONE, 12, e0176953.

Tzoulaki, I., Jarvelin, M.-R., Hartikainen, A.-L., Leinonen, M., Pouta, A., Paldanius, M., . . . Saikku, P. (2008). Size at birth, weight gain over the life course, and low-grade inflammation in young adulthood: Northern Finland 1966 Birth Cohort study. European Heart Journal, 29, 1049-1056.

van Otterdijk, S. D., Binder, A. M., \& Michels, K. B. (in press). Locus-specific DNA methylation in the placenta is associated with levels of pro-inflammatory proteins in cord blood and they are both independently affected by maternal smoking during pregnancy. Epigenetics.

Wadhwa, P. D., Buss, C., Entringer, S., \& Swanson, J. M. (2009). Developmental origins of health and disease: Brief history of the approach and current focus on epigenetic mechanisms. Seminars in Reproductive Medicine, 27, 358-368.

Weschsler, D., Golombok, S., \& Rust, J. (1992). WISC-III UK: Wechsler Intelligence Scale for Children. Sidcup, UK: Psychological Corporation.

Yoon, B. H., Romero, R., Park, J. S., Kim, M., Oh, S.-Y., Kim, C. J., \& Jun, J. K. (2000). The relationship among inflammatory lesions of the umbilical cord (funisitis), umbilical cord plasma interleukin 6 concentration, amniotic fluid infection, and neonatal sepsis. American Journal of Obstetrics and Gynecology, 183, 1124-1129.

Zhang, Y., Collier, F., Naselli, G., Saffery, R., Tang, M. L., Allen, K. J., . . . Vuillermin, P. (2016). Cord blood monocyte-derived inflammatory cytokines suppress IL-2 and induce nonclassic "TH2-type" immunity associated with development of food allergy. Science Translational Medicine, 8, 321ra328-321ra328. 